\title{
Sub-wavelength focusing of high intensities in microfibre tips
}

\author{
G. Brambilla, M. Ding, J.-Y. Ou, N. Sessions, Y. Jung \\ Optoelectronics Research Centre, University of Southampton \\ Southampton, SO17 1BJ, United Kingdom
}

\author{
O. Fenwick, F. Di Stasio, F. Cacialli \\ Department of Physics and Astronomy and London Centre for Nanotechnology, University College London, \\ London WC1E 6BT, United Kingdom
}

\begin{abstract}
J-L. Kou and F. Xu
College of Engineering and Applied Sciences and National Laboratory of Solid State Microstructures, Nanjing University, Nanjing 210093, P. R. China
\end{abstract}

\begin{abstract}
Sub-wavelength efficient intensity confinement has been demonstrated in nanostructured optical microfibre tips. Focus Ion Beam (FIB) milling was used to nanostructure goldcoated optical microfibre tips and form apertures at the apex. Simulations were carried out to optimize the device design. Enhanced transmission efficiency (higher than $10^{-2}$ ) was achieved in spot sizes of $\sim \lambda / \mathbf{1 0}$. Nanostructured microfibre tips have the potential for a number of applications including optical recording, photolithography and scanning near-field optical microscopy (SNOM).
\end{abstract}

\section{INTRODUCTION}

Many applications like optical data recording, imaging, photolithography and optical nanoprocessing require optical spot sizes as small as possible. Diffraction is generally accepted as the limiting factor for focusing. The minimum achievable spot size $(\omega)$ is related to the wavelength $(\lambda)$ of light and to the refractive index $(n)$ of the medium where light is focused and can be approximated by [1]:

$$
\omega_{0} \geq \frac{\lambda}{2 n}
$$

Several techniques have been proposed to overcome the diffraction limit, including metamaterials [2-4], plasmonics [5-7], longitudinal evanescent waves [8-10], high index contrast waveguides [11], photonic crystal fibers [12], tapered microtube [13] and microfibre arrays [14].

Optical fibre tips have been widely used to confine light to spot sizes of the order of $50 \mathrm{~nm}$ [15-19] in SNOMs, but their efficiency is typically low (often of the order of $10^{-5}$ or smaller) and cannot stand powers in excess of few $\mathrm{mW}$. Devices based on plasmonics have been proposed for efficient tight light confinement, but they always dealt with small powers and mostly in planar geometries. Plasmonics has been used in an apertureless silver-coated optical fibre tip to convert the radially polarized waveguide modes of the optical fibre taper tip into the plasmons propagating at the outer surface was proposed [20]. Extremely high transmission efficiency has been predicted; yet, apertureless optical fibre tips with high transmission efficiency have never been experimentally demonstrated $[10,21]$. In 2009 , plasmonics has been used in apertured tips to confine light to spots with sub-wavelength $(\lambda / 3)$ sizes in optical fibers and microfibres [22, 23]: the overall transmission efficiency improved by orders of magnitude. Yet, the use of plasmonics can be limited by two major issues: 1) the minimal spot size is limited by the radial component of the evanescent field, which becomes increasingly significant at small aperture sizes, and 2) the maximum power injected into the tip is limited by the extent of the field at the border between dielectric and metal, which can become exceedingly high and result in an instantaneous melting or evaporation of the metal coating.

Here efficient sub-wavelength light confinement at high powers is investigated by nanostructuring metal coated optical microfibre tips.

\section{SimUlations}

3D Finite Element Method (FEM) simulations were carried out using the commercial software COMSOL 4.1 Multiphysics to numerically solve Maxwell equations in the frequency domain and optimize the microfibre tip design with respect to its transmission efficiencies. The geometry of the modeled microfibre tip conical cut structure is shown in Fig. 1.

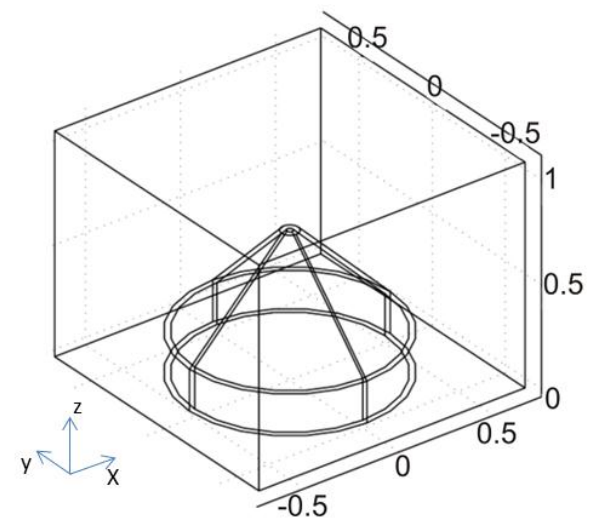

Figure 1. Schematic of optical microfibre tip geometry used in FEM modeling 
The microfibre tip is assumed to have a $1 \mu \mathrm{m}$ diameter and to be coated by a layer of gold with an aperture at its apex. The tip is surrounded by air in a box aimed to avoid reflections from boundaries. The chosen boundary conditions were: scattering boundary condition in the exterior boundary and continuity boundary condition in the interior boundary. Simulations were run with controlled mesh size $(50 \mathrm{~nm}$ in silica, $10 \mathrm{~nm}$ in gold coating and $100 \mathrm{~nm}$ in air surrounding) to make efficient use of computer memory. Circular polarization was chosen as launching condition for simulations as it is the most favorable polarization for applications in all-optical recording. Light wavelength was initially set to $\lambda=800 \mathrm{~nm}$. At the output the electric field was recorded on a plane $5 \mathrm{~nm}$ from the apex, as in optical recording, SNOM and photolithographic applications the working distance is of the order of few nanometers.

Fig. 2 shows the field in the microfibre tip for a geometry where plasmonics effects are significant. The tip has a slope of $\alpha=48.713^{\circ}$ and a $20 \mathrm{~nm}$ gold coating. A $30 \mathrm{~nm}$ circular aperture is open at the microfibre tip apex.

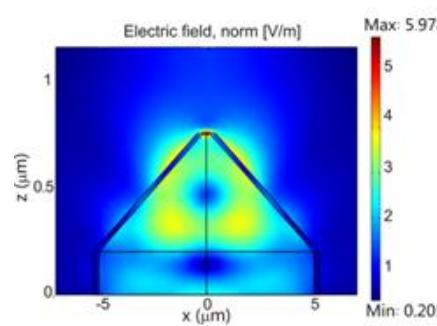

(a)

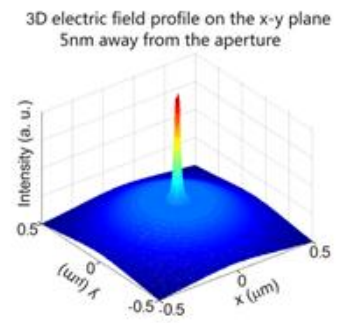

(c)

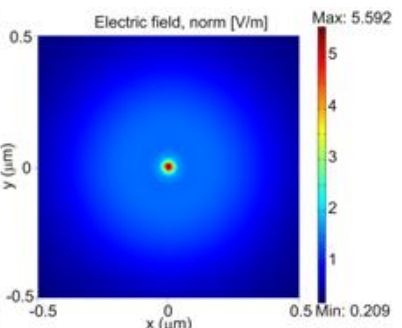

(b)

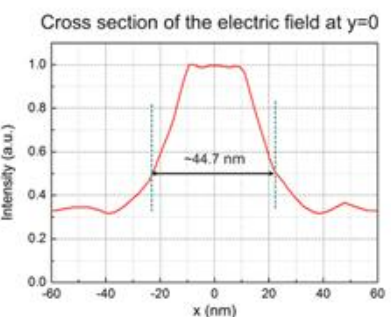

(d)
Figure 2. Electric field distribution in the microfibre tip coated with a $20 \mathrm{~nm}$ gold layer: (a) across the $x-z$ plane passing through the structure center; (b) across the $x-y$ plane, $5 \mathrm{~nm}$ above the aperture; (c) 3D distribution at the same $\mathrm{x}-\mathrm{y}$ plane; (d) cross section of the electric field intensity at $\mathrm{y}=0$. Aperture diameter and slope angle are $\mathrm{d}=30 \mathrm{~nm}$ and $\alpha=48.713^{\circ}$, respectively.

The field is extremely localized, with a full-width at half maximum (FWHM) of $44.7 \mathrm{~nm}$, i.e. $1 / 17$ of the incident wavelength. It is worth noting that the strong plasmonic radial evanescent field could limit the possibility to focus light; yet, in fig. 2(d) the extent of such a field is of the order of $30 \%$ of its maximum value.

Simulations with a smaller aperture were carried out to investigate whether this approach could lead to stronger confinement or it is inherently limited by the large radial evanescent field. Fig. 3 shows the electric field at $5 \mathrm{~nm}$ from the apex of a microfibre tip with a $5 \mathrm{~nm}$ aperture size. Although the small aperture induces a strong localization, for distances larger than $20 \mathrm{~nm}$ the evanescent field reaches considerable values and can exceed $50 \%$ of its maximum value.
Cross section of the electric field at $y=0$

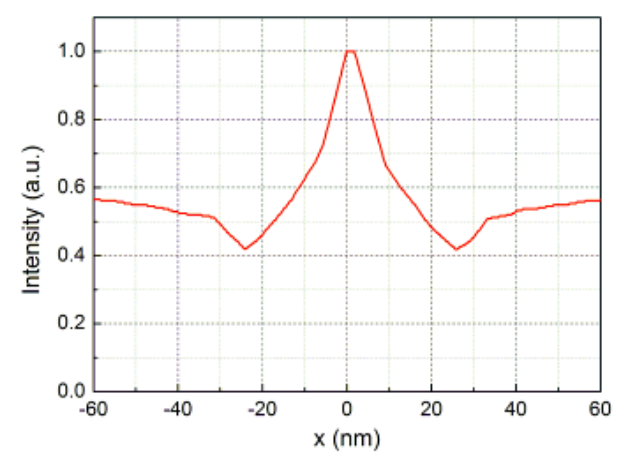

Figure 3. Electric field distribution in the microfibre tip coated with a $20 \mathrm{~nm}$ gold layer when the apex aperture is $5 \mathrm{~nm}$.

To evaluate the tip confinement properties, a transmission efficiency $\eta$ was defined as the absolute value of the ratio between the integral of the Poynting vector $S_{z}$ at the microfibre input and output apertures:

$$
\eta=\frac{\int_{\text {out }} S_{z} d A}{\int_{\text {in }} S_{z} d A}
$$

At the microfibre tip output only the section above the apex aperture was considered. $\eta$ was found to be orders of magnitude larger than that previously recorded for SNOM tips and to increase with decreasing microfibre tip slopes $\alpha$ and increasing aperture sizes [24]. For a $30 \mathrm{~nm}$ aperture, the efficiency increases from $\eta=0.765 \%$ at $\alpha=50^{\circ}$ to $\eta=2.06 \%$ at $\alpha=38^{\circ}$ and $\eta=4.90 \%$ at $\alpha=20^{\circ}$. Similarly, for a $5 \mathrm{~nm}$ aperture $\eta$ increases from $0.144 \%$ at $\alpha=50^{\circ}$ to $0.881 \%$ at $\alpha=35^{\circ}$ and $\eta=2.15 \%$ at $\alpha=20^{\circ}$. It is very important to note that also the overlap between the electric field and the metal decreases with decreasing $\alpha$. Fig. 4 shows electric field distributions across the $\mathrm{X}-\mathrm{z}$ plane passing through the structure center for different $\alpha$. While at $\alpha \sim 45^{\circ}$ there is a strong field at the metal - silica interface, at smaller angle the field is negligible.
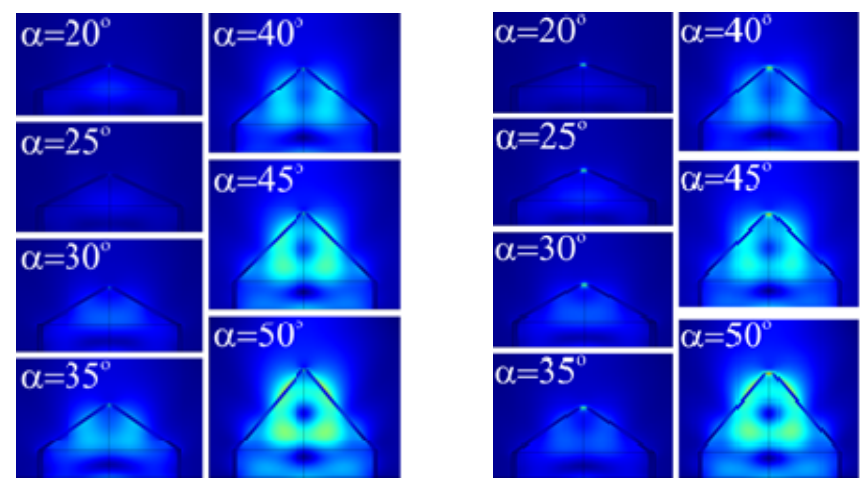

Figure 4. Electric field distribution across the $\mathrm{x}-\mathrm{z}$ plane passing through the structure center in the microfibre tip coated with a $20 \mathrm{~nm}$ gold layer when the apex aperture is 5 $\mathrm{nm}$ (left) and $30 \mathrm{~nm}$ (right).

Fig. 5 shows the field for a tip with a sharp slope $\left(\alpha=20^{\circ}\right)$ : light is well confined in a spot size $25 \mathrm{~nm}$ wide with only a small radial evanescent field outside the aperture region. 

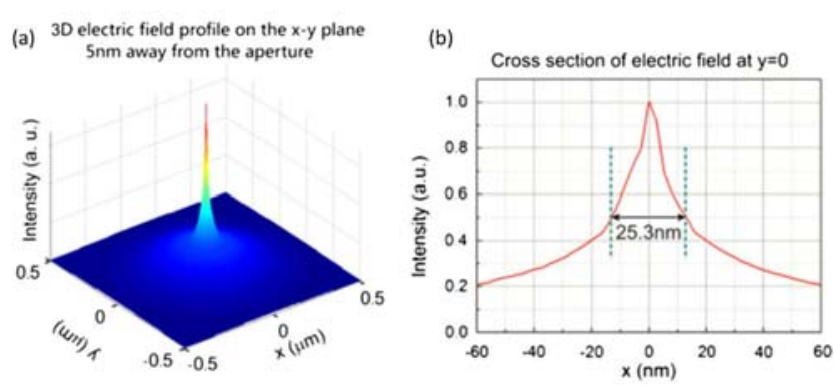

Figure 5. Electric field distribution in the microfibre tip coated with a $20 \mathrm{~nm}$ gold layer when the apex aperture is $5 \mathrm{~nm}$. (a) 3D distribution on the $\mathrm{x}-\mathrm{y}$ plane $5 \mathrm{~nm}$ distant from the apex. (b) Cross section of the electric field intensity at $y=0$.

\section{EXPERIMENTAL RESULTS}

Nanostructured microfibre tips with small apertures at their apex have been manufactured from conventional optical fibres in four main steps: (i) fabrication of optical microfibre tip, (ii) tip milling, (iii) surface metallization, and (iv) aperture formation.

A commercial pipette puller (P-2000, Sutter Instrument Inc., Novato, USA) was used to manufacture microfibers tip from a telecom single mode fibre (SMF-1300/1550-9/1250.25 -L (OZ optics, Canada) with $\sim 8.2 \mu \mathrm{m}$ core diameter, $\sim 125 \mu \mathrm{m}$ cladding diameter, $\sim 0.12$ numerical aperture and $1250 \mathrm{~nm}$ cut-off wavelength. As the main target of microfibre tip nanostructuring was high transmission efficiency, the taper angle in each point of the microfibre tip had to be small enough [25] to adiabatically convert the fundamental mode in the core into the fundamental mode in the microfibre. The pipette puller settings were optimized to provide a predetermined taper shape which guaranteed good adiabaticity.

Microfibre tips with an adiabatic profile were then nanostructured using a focused ion beam (FIB) milling system. FIB is a machining technique with resolution smaller than $10 \mathrm{~nm}$ and it allows for extremely high precision in the material removal. Although processing current was kept reasonably small to maintain high shaping precision, microfibre cleavage with specific angles required a short processing time, typically of the order of one minute.

Metallization with gold was then performed on the microfibre tips using a thermal evaporator. The deposition of a thin metal layer on the tip surface both allows for an enhanced light confinement and avoids charge accumulation during FIB machining. In fact, charging is a major issue as it degrades FIB resolution and it becomes extremely important when features of the order of $10 \mathrm{~nm}$ are investigated.

Finally, apertures were made on nanostructured samples using FIB. Two types of apertures were considered: the slot, achieved cutting the terminal end of the fibre tip, and the hole, made by drilling the tip along the longitudinal direction.

Fig. 6 shows scanning electron microscope (SEM) images of some fabricated apertures: fig. 6(a) and (b) have slot apertures, while 6(c) and (d) have holes. Fig. 6(c) shows the smallest aperture manufactured to date, which has a diameter of $\sim 13 \mathrm{~nm}$.

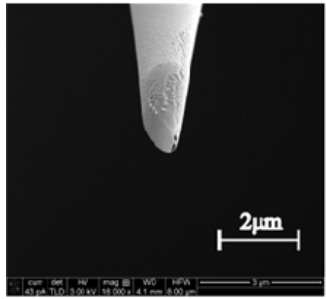

(घ)

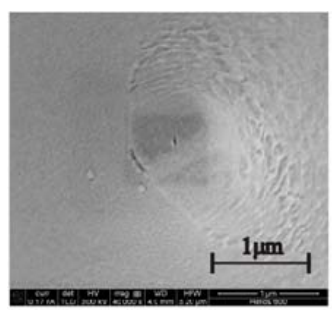

(c)

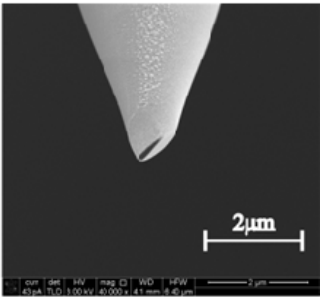

(b)

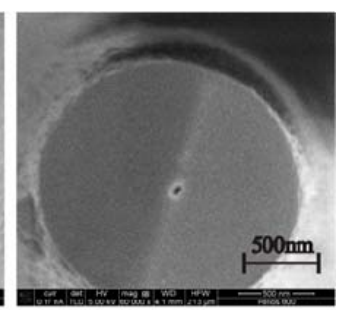

(d)
Figure 6. SEM micrographs of apertures opened at the gold-coated microfibre tip apexes. (a) Single-ramp microfibre tip with aperture size $\sim 66$ $\mathrm{nm}$; (b) wedge microfibre tip with max slit aperture size $\sim 84 \mathrm{~nm}$; (c,d) wedge tips with hole size (c) $\sim 13 \mathrm{~nm}$ and (d) $\sim 21 \mathrm{~nm}$.

The microfibre tip $\eta$ was measured injecting light from a supercontinuum source (Fianium, Southampton, UK) delivering 400 fs pulses over the wavelength range 450 $1800 \mathrm{~nm}$ into the samples under test. A modal filter [26] and polarization controller were inserted before the sample to provide a single mode propagation with specific polarization properties. A multimode fibre placed in closed proximity to the microfibre tip aperture was used to collect the transmitted light and deliver it to an optical spectrum analyzer (AQ6317, Yokogawa, Japan). Measurements without sample were performed to provide a normalization baseline for all spectra.

The sample showed in fig. 6(a) had a transmissivity of $\eta$ $\sim 5 \%$ at $\lambda=1100 \mathrm{~nm}$, where light is confined to a spot size as small as $\sim \lambda / 15$. The overall $\eta$ is orders of magnitude larger than that $\left(10^{-4} \sim 10^{-5}\right)$ recorded in SNOM tips of similar size.

It is also interesting to note that the supercontinuum source has a nominal power of $6 \mathrm{~W}$ and the power which was launched into the tips was of the order of a fraction of a Watt. No sign of degradation was observed.

\section{CONCLUSIONS}

In summary, preliminary studies on the efficient subwavelength confinement of light at high powers in apertured microfibre tips have been presented. Simulations predicted the possibility to focus light to $25 \mathrm{~nm}$ spot sizes with an efficiency of few percent and could provide a reference for the fabrication of microfibre tips suitable for data recording, imaging and photolithography. Experimentally, devices were manufactured using a pipette-puller, an evaporator, and a focused ion beam system. Apertures as small as $13 \mathrm{~nm}$ were fabricated and transmissivities of few percent recorded. The microfibre tips could stand powers as large as a fraction of a Watt with no significant degradation.

\section{ACKNOWLEDGMENT}

G.B. gratefully acknowledges the Royal Society (London, U.K.) for his University Research Fellowship. 


\section{REFERENCES}

[1] M. Born and E. Wolf, Principles of Optics: Electromagnetic Theory of Propagation, Interference and Diffraction of Light: Pergamon Press, 1999.

[2] N. Fang, H. Lee, C. Sun, and X. Zhang, "Subdiffraction-limited optical imaging with a silver superlens," Science, vol. 308, pp. 534-537, Apr 22 2005.

[3] A. Salandrino and N. Engheta, "Far-field subdiffraction optical microscopy using metamaterial crystals: Theory and simulations," Physical Review B, vol. 74, Aug 2006.

[4] V. M. Shalaev, "Optical negative-index metamaterials," Nature Photonics, vol. 1, pp. 41-48, Jan 2007.

[5] R. Zia, M. D. Selker, P. B. Catrysse, and M. L. Brongersma, "Geometries and materials for subwavelength surface plasmon modes," Journal of the Optical Society of America a-Optics Image Science and Vision, vol. 21, pp. 2442-2446, Dec 2004.

[6] S. A. Maier, P. G. Kik, H. A. Atwater, S. Meltzer, E. Harel, B. E. Koel, and A. A. G. Requicha, "Local detection of electromagnetic energy transport below the diffraction limit in metal nanoparticle plasmon waveguides," Nature Materials, vol. 2, pp. 229-232, Apr 2003.

[7] W. L. Barnes, A. Dereux, and T. W. Ebbesen, "Surface plasmon subwavelength optics," Nature, vol. 424, pp. 824-830, Aug 142003.

[8] E. Betzig and J. K. Trautman, "Near-Field Optics Microscopy, Spectroscopy, and Surface Modification Beyond the Diffraction Limit," Science, vol. 257, pp. 189-195, Jul 101992.

[9] H. Heinzelmann and D. W. Pohl, "Scanning nearField Optical Microscopy," Applied Physics aMaterials Science \& Processing, vol. 59, pp. 89101, Aug 1994.

[10] L. Novotny, D. W. Pohl, and B. Hecht, "Scanning near-Field Optical Probe with Ultrasmall Spot Size," Optics Letters, vol. 20, pp. 970-972, May 1 1995.

[11] Q. F. Xu, V. R. Almeida, R. R. Panepucci, and M. Lipson, "Experimental demonstration of guiding and confining light in nanometer-size lowrefractive-index material," Optics Letters, vol. 29, pp. 1626-1628, Jul 152004.

[12] G. S. Wiederhecker, C. M. B. Cordeiro, F. Couny, F. Benabid, S. A. Maier, J. C. Knight, C. H. B. Cruz, and H. L. Fragnito, "Field enhancement within an optical fibre with a subwavelength air core," Nature Photonics, vol. 1, pp. 115-118, Feb 2007.

[13] J. A. Fu, H. T. Dong, and W. Fang, "Subwavelength focusing of light by a tapered microtube," Applied Physics Letters, vol. 97, Jul 262010.

[14] X. Wang, J. Fu, X. Liu, and L. M. Tong, "Subwavelength focusing by a micro/nanofiber array," Journal of the Optical Society of America a-
Optics Image Science and Vision, vol. 26, pp. 18271833, Aug 2009.

B. Hecht, B. Sick, U. P. Wild, V. Deckert, R. Zenobi, O. J. F. Martin, and D. W. Pohl, "Scanning near-field optical microscopy with aperture probes: Fundamentals and applications," Journal of Chemical Physics, vol. 112, pp. 7761-7774, May 8 2000.

[16] J. A. Veerman, A. M. Otter, L. Kuipers, and N. F. van Hulst, "High definition aperture probes for near-field optical microscopy fabricated by focused ion beam milling," Applied Physics Letters, vol. 72, pp. 3115-3117, Jun 151998.

[17] L. Novotny, D. W. Pohl, and P. Regli, "LightPropagation through Nanometer-Sized Structures the 2-Dimensional-Aperture Scanning near-Field Optical Microscope," Journal of the Optical Society of America a-Optics Image Science and Vision, vol. 11, pp. 1768-1779, Jun 1994.

[18] D. W. Pohl and L. Novotny, "Near-Field Optics Light for the World of Nano," Journal of Vacuum Science \& Technology B, vol. 12, pp. 1441-1446, May-Jun 1994.

[19] R. Riehn, A. Charas, J. Morgado, and F. Cacialli, "Near-field optical lithography of a conjugated polymer," Applied Physics Letters, vol. 82, pp. 526528, Jan 272003.

[20] W. Ding, S. R. Andrews, and S. A. Maier, "Internal excitation and superfocusing of surface plasmon polaritons on a silver-coated optical fiber tip," Physical Review A, vol. 75, Jun 2007.

[21] T. J. Antosiewicz, P. Wrobel, and T. Szoplik, "Superfocusing on a Dielectric-Metal-Dielectric Apertureless Scanning Near-Field Optical Microscope Probe," Icton: 2009 11th International Conference on Transparent Optical Networks, Vols 1 and 2, pp. 554-557, 2009.

F. Renna, G. Brambilla, and D. C. Cox, "Light Confinement in Optical Fibers Using Surface Plasmon Polaritons," Ieee Photonics Technology Letters, vol. 21, pp. 1508-1510, Oct 152009.

F. Renna, D. Cox, and G. Brambilla, "Efficient subwavelength light confinement using surface plasmon polaritons in tapered fibers," Optics Express, vol. 17, pp. 7658-7663, Apr 272009.

O. F. M. Ding, F. Di Stasio, J. Ou, N. P. Sessions, Y. Jung, F. Cacialli, G. Brambilla, "Efficient Light Confinement with Nanostructured Optical Microfiber Tips," Optics Communications, 2012.

[25] J. D. Love, W. M. Henry, W. J. Stewart, R. J. Black, S. Lacroix, and F. Gonthier, "Tapered Single-Mode Fibers and Devices .1. Adiabaticity Criteria," Iee Proceedings-J Optoelectronics, vol. 138, pp. 343354, Oct 1991.

[26] Y. M. Jung, G. Brambilla, and D. J. Richardson, "Efficient higher-order mode filtering in multimode optical fiber based on an optical microwire," Aoe 2008: Asia Optical Fiber Communication and Optoelectronic Exposition and Conference, 2009. 